OPEN ACCESS

Edited by:

Cynthia J. Roberts,

The Ohio State University,

United States

Reviewed by:

Juan Alvarez De Toledo Elizalde,

Clínica Oftalmológica Barraquer, Spain

Soheila Asgari,

Noor Eye Hospital, Iran

*Correspondence:

Xingtao Zhou

doctzhouxingtao@163.com

tThese authors have contributed equally to this work and share first

authorship

Specialty section: This article was submitted to

Ophthalmology,

a section of the journal

Frontiers in Medicine

Received: 03 February 2021 Accepted: 11 June 2021

Published: 07 July 2021

Citation:

Jian W, Tian M, Zhang X, Sun L, Shen Y, Li M and Zhou X (2021)

One-Year Follow-Up of Corneal

Biomechanical Changes After Accelerated Transepithelial Corneal

Cross-Linking in Pediatric Patients

With Progressive Keratoconus.

Front. Med. 8:663494.

doi: 10.3389/fmed.2021.663494

\section{One-Year Follow-Up of Corneal Biomechanical Changes After Accelerated Transepithelial Corneal Cross-Linking in Pediatric Patients With Progressive Keratoconus}

\author{
Weijun Jian $^{1,2,3+}$, Mi Tian ${ }^{1,2,3 \dagger}$, Xiaoyu Zhang ${ }^{1,2,3}$, Ling Sun ${ }^{1,2,3}$, Yang Shen ${ }^{1,2,3}$, Meiyan Li $^{1,2,3}$ \\ and Xingtao Zhou ${ }^{1,2,3 *}$ \\ ${ }^{1}$ Department of Ophthalmology and Optometry, Eye and ENT Hospital, Fudan University, Shanghai, China, ${ }^{2}$ Key Laboratory \\ of Myopia, Chinese Academy of Medical Sciences, Shanghai, China, ${ }^{3}$ Shanghai Research Center of Ophthalmology and \\ Optometry, Shanghai, China
}

Aims: This study aimed to investigate the corneal biomechanical changes and topographic outcomes of accelerated transepithelial corneal cross-linking (ATE-CXL) in pediatric progressive keratoconus.

Methods: In this prospective longitudinal study, 31 eyes of 28 pediatric patients with keratoconus ( 21 boys and 7 girls; mean age, $14.35 \pm 2.68$ years) undergoing ATE-CXL (epithelium-on procedure with $45 \mathrm{~mW} / \mathrm{cm}^{2}$ for $320 \mathrm{~s}$ ) were included. Corvis ST was used to measure dynamic corneal response parameters at baseline and at 12 month after ATE-CXL. Corneal keratometry and corneal thickness were measured using Pentacam pre-operatively and 1, 6, and 12 month post-operatively.

Results: No serious complications occurred during or after ATE-CXL. The maximum keratometry values were $60.10 \pm 7.51 \mathrm{D}$ pre-operatively and $61.42 \pm 8.92,61.17 \pm$ 7.96, and $60.02 \pm 7.58 \mathrm{D}$ at 1,6 , and 12 month after ATE-CXL $(P>0.05)$, respectively. Corneal thickness remained stable during the 12-month follow-up $(P>0.05)$. At post-operative 12 month, first applanation time $(P<0.001)$, first applanation length $(P=0.004)$, second applanation velocity $(P=0.014)$, highest concavity time $(P=0.022)$, and radius of curvature at highest concavity $(P=0.031)$ increased significantly. The value of stiffness parameter at first applanation was significantly increased from $57.70 \pm 27.57$ pre-operatively to $63.36 \pm 27.09$ at 12 months after ATE-CXL $(P=0.018)$.

Conclusions: ATE-CXL is safe and effective in stabilizing the progression of pediatric keratoconus. Changes in corneal biomechanical response consistent with stiffening following ATE-CXL were observed in pediatric patients with keratoconus.

Keywords: pediatric, keratoconus, accelerated transepithelial cross-linking, Corvis ST, corneal biomechanics 


\section{PRECIS}

ATE-CXL is safe and effective in stabilizing the progression and the corneal biomechanical properties of pediatric keratoconus.

\section{INTRODUCTION}

Keratoconus is a bilateral progressive non-inflammatory ectatic corneal dystrophy characterized by thinning and steepening of the paracentral cornea (1), which usually appears during puberty, and early adulthood. Keratoconus seems to progress faster and to be more advanced at the time of diagnosis in pediatric patients compared to adults (2). Therefore, more attention should be paid to the treatment of pediatric keratoconus at early stages of the disease (3).

Corneal cross-linking (CXL) is considered an effective treatment for halting or reducing the progression of keratoconus with increasing corneal biomechanical stiffness. The majority of published studies (4-7) have demonstrated the safety and efficacy of CXL for pediatric keratoconus; however, most of these studies focused on conventional corneal cross-linking (CCXL; epithelium-off, $3 \mathrm{~mW} / \mathrm{cm}^{2}$ for $30 \mathrm{~min}$ ) in the corneas of pediatric patients. Accelerated transepithelial corneal crosslinking (ATE-CXL; epithelium-on, $45 \mathrm{~mW} / \mathrm{cm}^{2}$ for $320 \mathrm{~s}$ ) (811) has several benefits over the conventional method such as epithelium-on procedure, less treatment time, less intraoperative and post-operative complications, and greater comfort for the patients. We were the first to report [in a previous study $(10,11)]$ the long-term safety and efficacy of ATE-CXL for progressive pediatric keratoconus.

Abnormal corneal biomechanical behavior is the most probable etiologic factor for keratoconus, thereby resulting in corneal morphologic changes (12). According to previous studies, the reduction of corneal biomechanical properties plays an important role in the progression of keratoconus. Corvis ST $(13,14)$ (OCULUS Optikgeräte GmbH; Wetzlar, Germany) is a non-contact tonometer that uses an ultra-high speed Scheimpflug camera to directly observe the response of the cornea to an air pressure pulse and provides a series of dynamic corneal response (DCR) parameters related to cornea deformation and corneal stiffness in vivo.

Several published studies have reported the use of Corvis ST in assessing the biomechanical properties of the cornea and in the diagnosis and treatment of keratoconus $(15,16)$. A previous study (17) has suggested early changes in biomechanics following CXL by Corvis ST in adult patients with keratoconus. However, studies that assess the biomechanical properties before and after ATE-CXL in pediatric patients with keratoconus are still lacking. Therefore, the aim of this study was to investigate the corneal biomechanical changes and topographic outcomes of ATE-CXL in pediatric patients with progressive keratoconus.

\section{PATIENTS AND METHODS}

\section{Patients}

In this prospective study, we enrolled pediatric patients (aged 8-17 years) who were diagnosed with progressive keratoconus
TABLE 1 | Demographic data of the patients (Mean \pm SD).

\begin{tabular}{lc}
\hline & Mean \pm SD \\
\hline Age (year) & $14.35 \pm 2.68$ \\
Sphere (D) & $-6.04 \pm 5.31$ \\
Cylinder (D) & $-5.10 \pm 2.15$ \\
SE (D) & $-8.59 \pm 5.89$ \\
CDVA (logMAR) & $0.41 \pm 0.32$ \\
K1 (D) & $48.07 \pm 4.94$ \\
K2 (D) & $52.74 \pm 5.07$ \\
Kmax (D) & $60.10 \pm 7.51$ \\
CCT $(\mu \mathrm{m})$ & $479.10 \pm 45.33$ \\
AT $(\mu \mathrm{m})$ & $472.13 \pm 45.83$ \\
TCT $(\mu \mathrm{m})$ & $461.55 \pm 45.83$
\end{tabular}

D, diopters; SE, spherical equivalent; CDVA, corrected distance visual acuity; K1, steepest meridian keratometry; K2, flattest meridian keratometry; Kmax, maximum keratometry; CCT, central corneal thickness; AT, apex thickness; TCT, thinnest corneal thickness.

at the Eye and ENT Hospital of Fudan University in Shanghai, China. Evidence of progressive keratoconus included an increase in the maximum keratometry (Kmax) value or astigmatism $>1$ $\mathrm{D}$ in 1 year. Patients were excluded from the study if they had any of the following: stromal scarring in the cornea; worn rigid gas permeable lenses or soft contact lenses more than 4 or 2 weeks, respectively; a history of other ocular diseases that could affect corneal morphology; previous ocular surgeries; or allergy to riboflavin. We analyzed 31 eyes ( 24 boys and 7 girls, 14 right eyes and 17 left eyes) of 28 patients with a mean age of $14.35 \pm$ 2.68 years. The baseline characteristics of the study population are shown in Table $\mathbf{1 .}$

This study was approved by the Ethics Committee of the Eye and ENT Hospital of Fudan University and was carried out following the tenets of the Declaration of Helsinki. One parent or legal guardian of each subject was provided with informed consent after a detailed explanation of the procedure prior to treatment.

\section{Measurements}

The central corneal thickness (CCT), thinnest corneal thickness (TCT), apex thickness (AT), steepest meridian keratometry (K1), flattest meridian keratometry (K2), and Kmax values were obtained via Pentacam (OCULUS Optikgeräte GmbH; Wetzlar, Germany). All measurements with the Pentacam were operated by the same experienced technicians. The data were collected pre-operatively and at 1, 6, and 12 month after ATE-CXL.

DCR parameters were measured by the Corvis ST. The Corvis ST can capture series images of cornea by using the ultra-highspeed Scheimpflug camera while consistent air puff deforms the cornea. The definitions of the DCR parameters are listed in Table 2. Stiffness parameter at first applanation (SP-A1) was defined as the resultant pressure on the cornea divided by the corneal displacement in the first applanation position, which is considered to be a sensitive parameter for assessing corneal stiffness (18). A higher SP-A1 value indicates that the cornea has a stiffer response. All measurements with the Corvis ST were 
TABLE 2 | The meaning of Corvis ST parameters.

\begin{tabular}{|c|c|}
\hline Parameter & Explanation \\
\hline First applanation time (AT1) & Time to reach first applanation \\
\hline First applanation length (AL1) & Length of the deformed part of the cornea at first applanation \\
\hline First applanation velocity (AV1) & Corneal velocity at first applanation \\
\hline Second applanation time (AT2) & Time to reach second applanation \\
\hline Second applanation length (AL2) & Length of the deformed part of the cornea at second applanation \\
\hline Second applanation velocity (AV2) & Corneal velocity at second applanation \\
\hline Highest concavity time (HCT) & Time to reach highest concavity \\
\hline Deformation amplitude (DA) & Sagittal length of deformation of the apex at highest concavity \\
\hline Peak distance (PD) & The distance of the part of the cornea without deformation at highest concavity \\
\hline Radius of curvature (Rad) & Radius of curvature at highest concavity \\
\hline Deformation amplitude ratio (DAR) & The ratio between the deformation amplitude at the apex and the deformation amplitude at $2 \mathrm{~mm}$ \\
\hline Integrated radius (Integr Rad) & The integrated area under the inverse radius curve during the concave phase \\
\hline Stiffness parameter at first applanation (SP-A1) & $\begin{array}{l}\text { Pressure at first applanation-Biomechanically corrected intraocular pressure/deflection amplitude at first } \\
\text { applanation }\end{array}$ \\
\hline Ambrósio's relational thickness horizontal (ARTh) & $\begin{array}{l}\text { The division between corneal thickness at the thinnest point and the progression index that describes the } \\
\text { thickness increase from the thinnest point to the periphery }\end{array}$ \\
\hline
\end{tabular}

operated by the same experienced technicians. All patients were followed-up at post-operative 12 month.

\section{Cross-Linking Technique}

All operations were performed by the same surgeon (X Zhou). ATE-CXL was initiated under topical anesthesia using oxybuprocaine hydrochloride eye drops. The cornea was treated by using ParaCel solution (0.25\% riboflavin and benzalkonium chloride, Avedro) in the trephine (66 vision Tech, China) for $4 \mathrm{~min}$, and then Vibex Xtra solution (0.25\% riboflavin solution, Avedro) was dripped into the trephine for $6 \mathrm{~min}$. After that, the cornea was rinsed with a balanced salt solution. Subsequently, the cornea was then exposed to $370 \mathrm{~nm}$ ultraviolet-A (UVA) light with the Avedro's KXL System (Avedro, Inc) for $5 \mathrm{~min}$ and $20 \mathrm{~s}$ at an irradiance level of $45 \mathrm{~mW} / \mathrm{cm}^{2}$ in pulsed mode $(1 \mathrm{~s}$ on, $1 \mathrm{~s}$ off). A balanced salt solution was applied to protect the cornea from dehydration during the irradiation. At the end of the procedure, the eye was patched with a bandage contact lens, and the contact lens was removed after 3 days until epithelial healing was complete. Post-operatively, $0.1 \%$ fluorometholone was prescribed seven times per day, and the frequency was gradually tapered down over 2 weeks. Additionally, topical antibiotic (levofloxacin) and artificial tears were prescribed for four times per day for a week and month, respectively.

\section{Data Analysis}

Statistical analysis was performed in SPSS software (version 23; SPSS, Inc., Chicago, IL, USA) and SAS (version 9.4; SAS Institute, Cary, NC, USA). The results were analyzed using the Wilcoxon rank-sum test and repeated measures analyses of variance with Bonferroni-adjusted post-hoc comparisons. Inter-eye correlation was adjusted using a linear mixed model with random intercept to compare preoperative and post-operative parameters. Since certain DCR parameters were influenced by intraocular pressure (IOP) (19), it was included as a covariate in the model. Each follow-up result was compared with the preoperative values. $P$ $<0.05$ was considered statistically significant.

\section{RESULTS}

All surgical procedures were completed successfully without any intraoperative or post-operative complications.

\section{Corneal Keratometry}

Pre- and post-operative corneal keratometry values are presented in Figure 1. The Kmax values were $60.10 \pm 7.51 \mathrm{D}$ preoperatively, and $61.42 \pm 8.92,61.17 \pm 7.96,60.02 \pm 7.58 \mathrm{D}$ at 1,6 , and 12 month after the operation. All patients showed no significant $(P>0.05)$ changes in $\mathrm{K} 1, \mathrm{~K} 2$, and $\mathrm{Kmax}$ values in comparison to preoperative values at each follow-up time point.

\section{Corneal Thickness}

Figure 2 shows the preoperative and post-operative corneal thickness. The CCT values were $479.10 \pm 45.33 \mu \mathrm{m}$ preoperatively, and $476.90 \pm 43.83,474.63 \pm 43.28$, and $479.10 \pm 41.61 \mu \mathrm{m}$ at 1,6 , and 12 month after the operation, respectively. No statistically significant $(P>0.05)$ changes were found for CCT, TCT, and AT values during the 1-year follow-up.

\section{DCR Parameters}

The results of the corneal biomechanical properties assessment using the Corvis ST are illustrated in Table 3. The first applanation time (AT1), first applanation length (AL1), second applanation velocity (AV2), highest concavity time (HCT), and radius of curvature (Rad) increased significantly $(P<0.001$, $P=0.004, P=0.014, P=0.022$, and $P=0.031$, respectively) in 1 year after ATE-CXL. Furthermore, The SP-A1 value significantly increased from $57.70 \pm 27.57$ preoperatively to $63.36 \pm 27.09$ at 1 year post-operatively $(P=0.018)$. There were no significant differences in other parameters between the preoperative and post-operative time points $(P>0.05)$. 


\section{$\mathrm{K}$ value (D)}

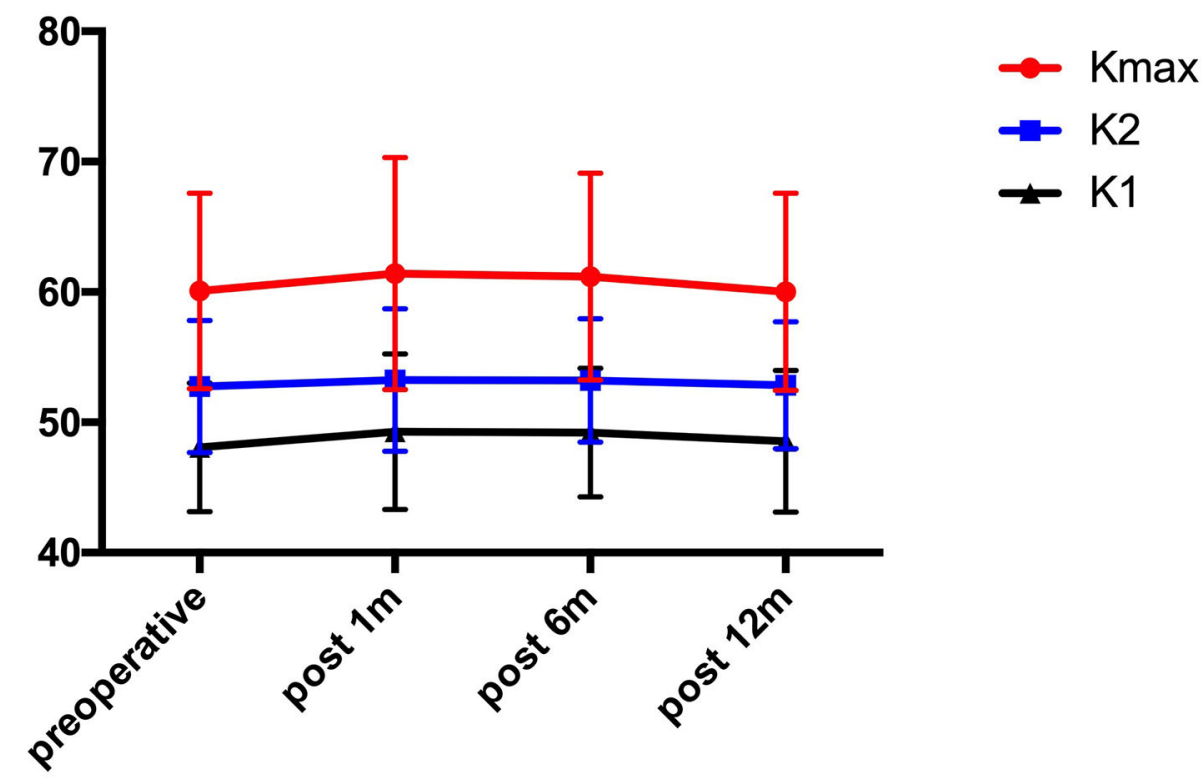

time points

FIGURE 1 | K1, K2, and Kmax before and after ATE-CXL during 1-year follow-up. There was no statistically significant $(P>0.05)$ alteration at each visit in comparison to preoperative values. (K1, steepest meridian keratometry; K2, flattest meridian keratometry; Kmax, maximum keratometry; ATE-CXL, accelerated transepithelial corneal cross-linking).

\section{DISCUSSION}

CXL is an effective treatment widely used to treat keratoconus. It is important to evaluate the efficacy of CXL by examination after the procedure, especially for pediatric patients with keratoconus because of the fast visual-developing period and the fact that any pathogenic effects may lead the irreversible vision loss. Corvis ST can be used to provide a series of DCR parameters in the diagnosis and treatment assessment of patients with keratoconus. In this study, we are the first to investigate the changes of corneal biomechanical properties in pediatric patients with progressive keratoconus after ATE-CXL.

Our results showed that the eyes remained stable with respect to Kmax 1 year after ATE-CXL when compared to the preoperative assessment, which may indicate that ATE-CXL is an effective treatment for halting the progression of pediatric keratoconus. Or et al. (7) and Eissa et al. (20) reported that Kmax remained stable 1 year after C-CXL and accelerated CXL (A-CXL) for pediatric patients with keratoconus, which was consistent with the results of our study.

The obtained results suggested no significant changes in CCT, TCT, and AT 1 year after ATE-CXL, which indicated the efficacy of stabilizing the corneal thickness in pediatric progressive keratoconus. Previous studies $(6,7,21)$ assessing the C-CXL outcomes in pediatric patients with keratoconus reported a significant decrease in corneal thickness compared with preoperative results. In our study, the corneal thickness remained stable at each follow-up examination because the corneal epithelium was preserved during the operation.

In this study, no intraoperative or post-operative complications were observed during the follow-up, which indicated that ATE-CXL can be safely used to treat pediatric patients. It has been reported that (22-24) complications, such as corneal haze or infection were found in a short follow-up period after C-CXL treatment for keratoconus, but these complications did not occur in our study. The UVA radiation energy of the CCXL treatment is lower, so the irradiation time is longer, and the patients need a longer recovery time due to the debridement of corneal epithelium. However, in this study, the process of ATECXL with epithelium-on procedure used higher UVA radiation energy, thus reducing the irradiation time, which not only allows patients to feel more comfortable during the treatment, but also let patients have shorter recovery periods after the treatment. Therefore, ATE-CXL is more suitable for pediatric patients with poor co-operation. Previous studies have postulated that A-CXL protocols with higher intensity UVA would result in more rapid oxygen depletion thereby reducing efficacy $(25,26)$. All eyes treated in our study received an application of pulsed light ATE-CXL, where oxygen availability theoretically should be greater than in the non-pulsed CXL treatments $(27,28)$. Mazzotta et al. (29) confirmed that pulsed light CXL treatment could produce better functional outcomes and a deeper stromal penetration than continuous light for keratoconus. 


\section{Corneal thickness $(\mu \mathrm{m})$}

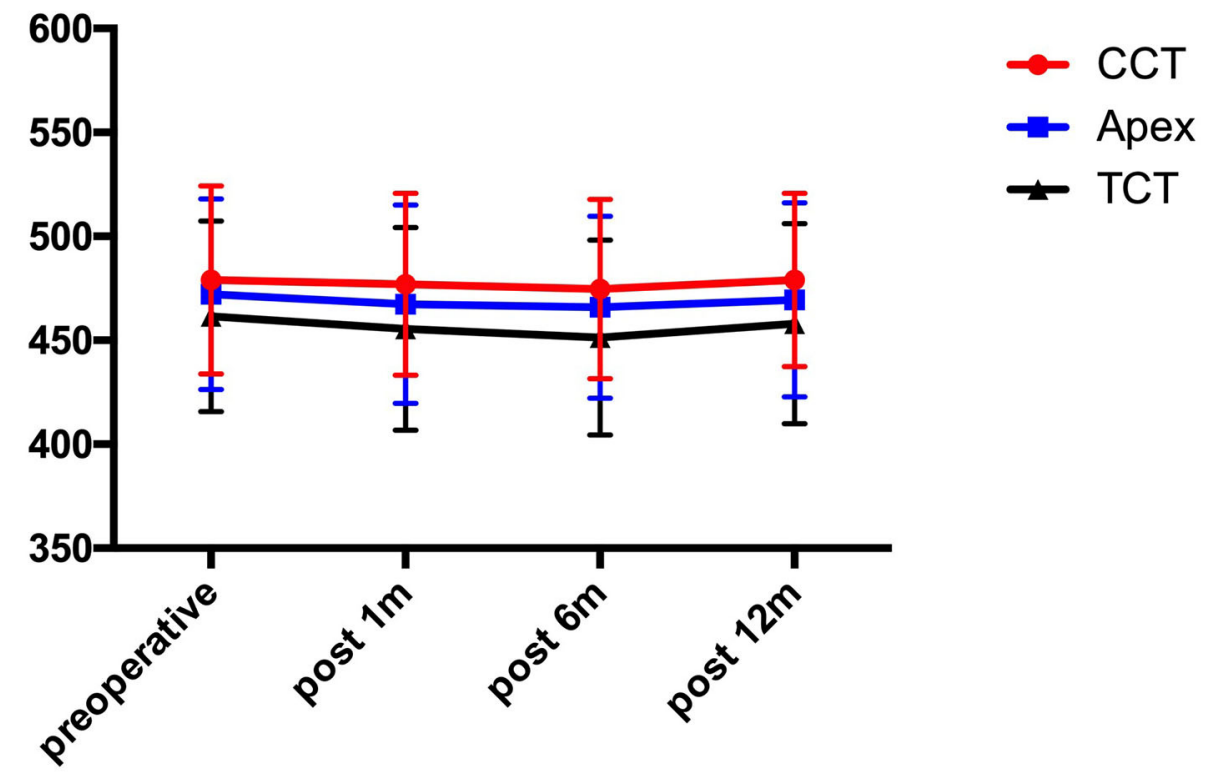

time points

FIGURE 2 | CCT, TCT, and AT before and after ATE-CXL during 1-year follow-up. No significant $(P>0.05)$ changes were found in CCT, TCT, and AT in comparison to preoperative values at each time point. (CCT, central corneal thickness; TCT, thinnest corneal thickness; AT, apex thickness; ATE-CXL, accelerated transepithelial corneal cross-linking).

Our comparative analysis of the corneal biomechanical parameters obtained before and after ATE-CXL showed that AT1 and AL1 increased significantly during 1-year follow-up. Vinciguerra et al. (17) reported that AT1 increased from 6.74 \pm 0.22 to $6.98 \pm 0.54$ in 34 eyes at 6 month after C-CXL, and Sedaghat et al. (30) reported that AL1 increased from $1.72 \pm$ 0.33 to $2.08 \pm 0.33$ in 18 eyes after C-CXL in a 4 -year followup study. However, these studies enrolled adult patients with keratoconus. AT1 represents the time when the cornea reached the first applanation. A stiffer cornea requires more time to reach the first applanation, so that the increase in AT1 indicates a significant increase in corneal stiffness. In this study, there was no change in DA at 1 year after ATE-CXL. DA represents the total displacement of the cornea from the original position at the highest corneal concavity time, so that the lower value represented the stronger resistance of cornea. Tomita et al. (31) found that DA significantly decreased in adult keratoconus after C-CXL. However, in a study of C-CXL by Sedaghat et al. (30) DA increased significantly in adult keratoconus at 4 years post-operatively, which indicated that our results needed to be confirmed in a longer follow-up. In addition, this study found that HCT increased significantly by 1 year after the operation, also suggesting the increase of corneal stiffness. HCT represents the time when the cornea reached the highest concavity, and healthier and stiffer corneas can more easily resist outside forces. Therefore, compared with eyes with keratoconus, a normal cornea needs more time to reach the highest concavity (32).
The deformation amplitude of a stiffer cornea is lower after being impacted by the pulse air, so the SP-A1 value is higher as detected by Corvis ST. Therefore, a healthy cornea shows a higher SP-A1 value compared with those with keratoconus (18). Another study also reported lower SP-A1 values in patients with more severe keratoconus (33). This study found that SP-A1 value increased significantly at 1year post-operatively, which suggested that ATE-CXL can be used to increase corneal stiffness for pediatric keratoconus. In the observation of 66 adult cases of keratoconus Vinciguerra et al. (34) also found that SP-A1 was significantly increased at 1 month after CXL. In addition, the integrated inverse radius was also considered as a parameter for the evaluation of corneal stiffness, and it was lower in healthy corneas compared to corneas with keratoconus (35). In this study, there was no change in Integr Rad value at 1 year after ATE-CXL. However, Hashemi et al. (36) found that the Integr Rad significantly decreased in a 2-year follow-up study of 37 eyes after A-CXL. The possible reason is that the severity of keratoconus enrolled in our study is relatively higher, with the preoperative Kmax value is larger than that of the above-mentioned study $(<55 \mathrm{D})$. To our knowledge, this is the first study to report changes in corneal biomechanical properties of pediatric patients with keratoconus who were treated with ATE-CXL, which indicated that ATECXL could enhance the biomechanical strength of the cornea in pediatric keratoconus. 
TABLE 3 | Pre-operative and post-operative parameters of Corvis ST (Mean \pm SD).

\begin{tabular}{lccc}
\hline & preoperative & $\mathbf{1 2}$ month & $\boldsymbol{P}$ value \\
\hline AT1 & $6.56 \pm 0.40$ & $6.88 \pm 0.34$ & $<0.001^{*}$ \\
AL1 & $1.85 \pm 0.24$ & $2.04 \pm 0.37$ & $0.004^{*}$ \\
AV1 & $0.17 \pm 0.03$ & $0.18 \pm 0.03$ & 0.809 \\
AT2 & $21.82 \pm 0.59$ & $21.90 \pm 0.68$ & 0.525 \\
AL2 & $1.53 \pm 0.42$ & $1.56 \pm 0.37$ & 0.812 \\
AV2 & $-0.33 \pm 0.08$ & $-0.31 \pm 0.13$ & $0.014^{*}$ \\
HCT & $16.47 \pm 0.51$ & $16.84 \pm 0.70$ & $0.022^{*}$ \\
PD & $4.97 \pm 0.47$ & $5.01 \pm 0.26$ & 0.465 \\
Rad & $4.64 \pm 0.95$ & $4.97 \pm 0.86$ & $0.031^{*}$ \\
DA & $1.27 \pm 0.22$ & $63.36 \pm 27.09$ & 0.097 \\
SP-A1 & $57.70 \pm 27.57$ & $198.05 \pm 90.23$ & 0.019 \\
ARTH & $210.12 \pm 90.94$ & $6.58 \pm 1.87$ & 0.168 \\
DAR & $6.44 \pm 1.53$ & $13.27 \pm 2.92$ & 0.381 \\
Integr Rad & $13.79 \pm 3.12$ & 0.062
\end{tabular}

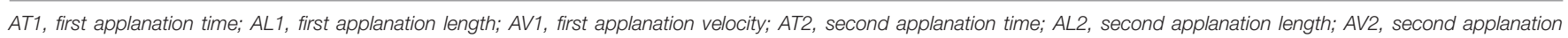

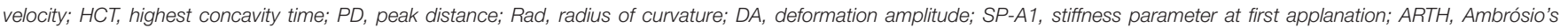
relational thickness horizontal; DAR, deformation amplitude ratio; Integr Rad, integrated radius.

*Statistically significant.

There were some limitations in this study. One was a relatively low number of patients, and another was the lack of a control group undergoing C-CXL. In addition, 1-year results of ATECXL is a short-term follow-up for pediatric patients. Further studies with a longer follow-up period to confirm the longterm biomechanical changes of the ATE-CXL treatment are also needed.

Our study suggests that ATE-CXL is a safe and effective treatment for pediatric patients with progressive keratoconus. The DCR parameters provided by Corvis ST can be used to evaluate changes of corneal biomechanics after cross-linking, which indicated that the corneal stiffness of pediatric keratoconus increased at 1 year after ATE-CXL, but the long-term effects need further observation.

\section{DATA AVAILABILITY STATEMENT}

The raw data supporting the conclusions of this article will be made available by the authors, without undue reservation.

\section{ETHICS STATEMENT}

The studies involving human participants were reviewed and approved by Ethics Committee of the Eye and ENT Hospital of

\section{REFERENCES}

1. Rabinowitz YS. Keratoconus. Surv Ophthalmol. (1998) 42:297319. doi: 10.1016/S0039-6257(97)00119-7

2. Vinciguerra $\mathrm{P}$, Albe E, Frueh BE, Trazza S, Epstein D. Two-year corneal cross-linking results in patients younger than 18 years with documented progressive keratoconus. Am J Ophthalmol. (2012) 154:5206. doi: 10.1016/j.ajo.2012.03.020
Fudan University. Written informed consent to participate in this study was provided by the participants' legal guardian/next of kin.

\section{AUTHOR CONTRIBUTIONS}

WJ, MT, and XZha were responsible for the initial plan, study design, data collection, data extraction, data interpretation, manuscript drafting, statistical analysis, and conducting the study. LS, YS, and ML were responsible for data collection, extraction, and critical revisions of the manuscript. WJ, MT, and XZho were responsible for data interpretation, manuscript drafting, supervision, and critical revisions of the manuscript for important intellectual content. XZho was the guarantor for this article and has full responsibility for this study. All authors contributed to the article and approved the submitted version.

\section{FUNDING}

This work was supported by grants from the National Natural Science Foundation of China (Grant No. 81770955); Joint research project of new frontier technology in municipal hospitals (SHDC12018103); the National Natural Science Foundation of China for Young Scholars (Grant No. 11702063).
3. Leoni-Mesplie S, Mortemousque B, Touboul D, Malet F, Praud D, Mesplie N, et al. Scalability and severity of keratoconus in children. Am J Ophthalmol. (2012) 154:56-62 el. doi: 10.1016/j.ajo.2012.01.025

4. Chatzis N, Hafezi F. Progression of keratoconus and efficacy of pediatric [corrected] corneal collagen cross-linking in children and adolescents. $J$ Refract Surg. (2012) 28:753-8. doi: 10.3928/1081597X-20121011-01

5. Viswanathan D, Kumar NL, Males JJ. Outcome of corneal collagen crosslinking for progressive keratoconus in paediatric 
patients. Biomed Res Int. (2014) 2014:140461. doi: 10.1155/2014/ 140461

6. Kumar Kodavoor S, Arsiwala AZ, Ramamurthy D. One-year clinical study on efficacy of corneal cross-linking in Indian children with progressive keratoconus. Cornea. (2014) 33:919-22. doi: 10.1097/ICO.0000000000000197

7. Or L, Rozenberg A, Abulafia A, Avni I, Zadok D. Corneal cross-linking in pediatric patients: evaluating treated and untreated eyes-5-year follow-up results. Cornea. (2018) 37:1013-7. doi: 10.1097/ICO.0000000000001629

8. Shen Y, Jian W, Sun L, Li M, Han T, Son J, et al. One-year follow-up of changes in corneal densitometry after accelerated $(45 \mathrm{~mW} / \mathrm{cm} 2)$ transepithelial corneal collagen cross-linking for keratoconus: a retrospective study. Cornea. (2016) 35:1434-40. doi: 10.1097/ICO.0000000000000934

9. Zhang X, Sun L, Chen Y, Li M, Tian M, Zhou X. One-year outcomes of pachymetry and epithelium thicknesses after accelerated $(45 \mathrm{~mW} / \mathrm{cm}(2))$ transepithelial corneal collagen cross-linking for keratoconus patients. Sci Rep. (2016) 6:32692. doi: 10.1038/srep32692

10. Tian M, Jian W, Sun L, Shen Y, Zhang X, Zhou X. One-year followup of accelerated transepithelial corneal collagen cross-linking for progressive pediatric keratoconus. BMC Ophthalmol. (2018) 18:75. doi: 10.1186/s12886-018-0739-9

11. Tian M, Jian W, Zhang X, Sun L, Zhou X. Three-year follow-up of accelerated transepithelial corneal cross-linking for progressive paediatric keratoconus. $\mathrm{Br}$ J Ophthalmol. (2020) 104:1608-12.

12. Scarcelli G, Besner S, Pineda R, Yun SH. Biomechanical characterization of keratoconus corneas ex vivo with Brillouin microscopy. Invest Ophthalmol Vis Sci. (2014) 55:4490-5. doi: 10.1167/iovs.14-14450

13. Sinha Roy A, Dupps WJ Jr. Patient-specific computational modeling of keratoconus progression and differential responses to collagen cross-linking. Invest Ophthalmol Vis Sci. (2011) 52:9174-87. doi: 10.1167/iovs.11-7395

14. Roberts CJ, Dupps WJ Jr. Biomechanics of corneal ectasia and biomechanical treatments. J Cataract Refract Surg. (2014) 40:991-8. doi: 10.1016/j.jcrs.2014.04.013

15. Ali NQ, Patel DV, McGhee CN. Biomechanical responses of healthy and keratoconic corneas measured using a noncontact scheimpflug-based tonometer. Invest Ophthalmol Vis Sci. (2014) 55:3651-9. doi: 10.1167/iovs.13-13715

16. Mercer RN. Waring GOt, Roberts CJ, Jhanji V, Wang Y, Filho JS, et al. Comparison of corneal deformation parameters in keratoconic and normal eyes using a non-contact tonometer with a dynamic ultra-high-speed Scheimpflug camera. J Refract Surg. (2017) 33:625-31. doi: 10.3928/1081597X-20170621-03

17. Vinciguerra R, Romano V, Arbabi EM, Brunner M, Willoughby CE, Batterbury $\mathrm{M}$, et al. In vivo early corneal biomechanical changes after corneal cross-linking in patients with progressive keratoconus. J Refract Surg. (2017) 33:840-6. doi: 10.3928/1081597X-20170922-02

18. Roberts CJ, Mahmoud AM, Bons JP, Hossain A, Elsheikh A, Vinciguerra R, et al. Introduction of two novel stiffness parameters and interpretation of air puff-induced biomechanical deformation parameters with a dynamic Scheimpflug analyzer. J Refract Surg. (2017) 33:266-73. doi: 10.3928/1081597X-20161221-03

19. Vinciguerra R, Elsheikh A, Roberts CJ, Ambrosio R Jr, Kang DS, Lopes BT, et al. Influence of pachymetry and intraocular pressure on dynamic corneal response parameters in healthy patients. J Refract Surg. (2016) 32:55061. doi: 10.3928/1081597X-20160524-01

20. Eissa SA, Badr Eldin N, Nossair AA, Ewais WA. Primary outcomes of accelerated epithelium-off corneal cross-linking in progressive keratoconus in children: a 1-year prospective study. J Ophthalmol. (2017) 2017:1923161. doi: 10.1155/2017/1923161

21. Sarac O, Caglayan M, Uysal BS, Uzel AGT, Tanriverdi B, Cagil N. Accelerated versus standard corneal collagen cross-linking in pediatric keratoconus patients: 24 months follow-up results. Cont Lens Anterior Eye. (2018) 41:4427. doi: 10.1016/j.clae.2018.06.001

22. Koller T, Mrochen M, Seiler T. Complication and failure rates after corneal crosslinking. J Cataract Refract Surg. (2009) 35:1358-62. doi: 10.1016/j.jcrs.2009.03.035

23. Knutsson KA, Paganoni G, Matuska S, Ambrosio O, Ferrari G, Zennato A, et al. Corneal collagen cross-linking in paediatric patients affected by keratoconus. $\mathrm{Br} J$ Ophthalmol. (2018) 102:248-52. doi: 10.1136/bjophthalmol-2016-310108
24. Kim BZ, Jordan CA, McGhee CN, Patel DV. Natural history of corneal haze after corneal collagen crosslinking in keratoconus using Scheimpflug analysis. J Cataract Refract Surg. (2016) 42:1053-9. doi: 10.1016/j.jcrs.2016.04.019

25. Brittingham S, Tappeiner C, Frueh BE. Corneal cross-linking in keratoconus using the standard and rapid treatment protocol: differences in demarcation line and 12-month outcomes. Invest Ophthalmol Vis Sci. (2014) 55:83716. doi: $10.1167 /$ iovs.14-15444

26. Touboul D, Efron N, Smadja D, Praud D, Malet F, Colin J. Corneal confocal microscopy following conventional, transepithelial, and accelerated corneal collagen cross-linking procedures for keratoconus. J Refract Surg. (2012) 28:769-76. doi: 10.3928/1081597X-20121016-01

27. Aldahlawi NH, Hayes S, O'Brart DP, Meek KM. Standard versus accelerated riboflavin-ultraviolet corneal collagen crosslinking: resistance against enzymatic digestion. J Cataract Refract Surg. (2015) 41:1989-96. doi: 10.1016/j.jcrs.2015.10.004

28. Aldahlawi NH, Hayes S, O'Brart DP, Akhbanbetova A, Littlechild SL, Meek KM. Enzymatic resistance of corneas crosslinked using riboflavin in conjunction with low energy, high energy, and pulsed UVA irradiation modes. Invest Ophthalmol Vis Sci. (2016) 57:1547-52. doi: 10.1167/iovs.15-18769

29. Mazzotta C, Traversi C, Paradiso AL, Latronico ME, Rechichi M. Pulsed light accelerated crosslinking versus continuous light accelerated crosslinking: one-year results. J Ophthalmol. (2014) 2014:604731. doi: 10.1155/2014/604731

30. Sedaghat MR, Momeni-Moghaddam H, Ambrosio R Jr, Roberts CJ, Yekta AA, Danesh Z, et al. Long-term evaluation of corneal biomechanical properties after corneal cross-linking for keratoconus: a 4-Year longitudinal study. $J$ Refract Surg. (2018) 34:849-56. doi: 10.3928/1081597X-20181012-02

31. Tomita M, Mita M, Huseynova T. Accelerated versus conventional corneal collagen crosslinking. J Cataract Refract Surg. (2014) 40:1013-20. doi: 10.1016/j.jcrs.2013. 12.012

32. Bak-Nielsen S, Pedersen IB, Ivarsen A, Hjortdal J. Dynamic Scheimpflugbased assessment of keratoconus and the effects of corneal cross-linking. $J$ Refract Surg. (2014) 30:408-14. doi: 10.3928/1081597X-20140513-02

33. Zhao Y, Shen Y, Yan Z, Tian M, Zhao J, Zhou X. Relationship among corneal stiffness, thickness, and biomechanical parameters measured by Corvis ST, Pentacam and ORA in keratoconus. Front Physiol. (2019) 10:740. doi: 10.3389/fphys.2019.00740

34. Vinciguerra R, Tzamalis A, Romano V, Arbabi EM, Batterbury M, Kaye SB. Assessment of the association between in vivo corneal biomechanical changes after corneal cross-linking and depth of demarcation line. J Refract Surg. (2019) 35:202-6. doi: 10.3928/1081597X-20190124-01

35. Fernandez J, Rodriguez-Vallejo M, Martinez J, Tauste A, Salvestrini P, Pinero DP. New parameters for evaluating corneal biomechanics and intraocular pressure after small-incision lenticule extraction by Scheimpflugbased dynamic tonometry. J Cataract Refract Surg. (2017) 43:80311. doi: 10.1016/j.jcrs.2017.03.035

36. Hashemi H, Ambrosio R Jr, Vinciguerra R, Vinciguerra P, Roberts CJ, Ghaffari R, et al. Two-year changes in corneal stiffness parameters after accelerated corneal cross-linking. J Biomech. (2019) 93:209-12. doi: 10.1016/j.jbiomech.2019.06.011

Disclaimer: This article's contents were solely the responsibility of the authors.

Conflict of Interest: The authors declare that the research was conducted in the absence of any commercial or financial relationships that could be construed as a potential conflict of interest.

The handling editor is a consultant for the manufacturer Corvis ST, and declares that the assessment of the manuscript was conducted in the absence of any commercial or financial relationships that could be construed as a potential conflict of interest.

Copyright (C) 2021 Jian, Tian, Zhang, Sun, Shen, Li and Zhou. This is an open-access article distributed under the terms of the Creative Commons Attribution License (CC $B Y)$. The use, distribution or reproduction in other forums is permitted, provided the original author(s) and the copyright owner(s) are credited and that the original publication in this journal is cited, in accordance with accepted academic practice. No use, distribution or reproduction is permitted which does not comply with these terms. 